\title{
Magnetic Properties of Bacterial Nanoparticles
}

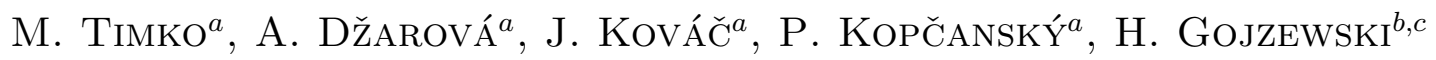 \\ AND A. SZlafereK ${ }^{d}$ \\ ${ }^{a}$ Institute of Experimental Physics, Slovak Academy of Sciences \\ Watsonova 47, 04001 Košice, Slovakia \\ ${ }^{b}$ Institute of Physics, Poznań University of Technology \\ Nieszawska 13A, 60-965 Poznań, Poland \\ ${ }^{c}$ Max Planck Institute for Polymer Research \\ Ackermannweg 10, 55128 Mainz, Germany \\ ${ }^{d}$ Institute of Molecular Physics, Polish Academy of Sciences \\ M. Smoluchowskiego 17, 60-179 Poznań, Poland
}

\begin{abstract}
The objective of this study is to prepare and study magnetic properties of biological magnetic nanoparticles (magnetosomes) as a product of biomineralization process of magnetotactic bacteria Magnetospirillum sp. AMB-1. From temperature dependence of remanent magnetization and coercive field the Verwey transition is clearly seen at $105 \mathrm{~K}$ as a consequence of the large anisotropy along the chains of magnetosomes.
\end{abstract}

PACS numbers: 47.65.Cb, 51.60.+a, 75.30.Gw, 75.50.Tt, 75.60.Nt, 75.60.Ej, 75.75.+a

\section{Introduction}

It is known that upon cooling below $T_{\mathrm{v}} \approx 120 \mathrm{~K}$ magnetite undergoes a sharp first-order metal-insulator transition (so-called Verwey transition) at which heat capacity has anomalous behavior, the resistivity increases sharply by two orders of magnitude [1] and transformation from cubic to monoclinic structure appears [2]. The results from measurements of remanence and coercive force measured in the monoclinic phase (below $T_{\mathrm{v}}$ ) for fine grained magnetites with medium particle sizes of $0.037,0.10$ and $0.22 \mu \mathrm{m}$ have showed that coercive force $H_{\mathrm{c}}$ and remanence magnetization increase on cooling through $T_{\mathrm{v}}$ in submicron magnetite particles [3]. These increases reflect the large increase in magnetocrystalline anisotropy and magnetostriction in the cubic $\rightarrow$ monoclinic transformation.

It is well known that bacterial magnetite nanoparticles (magnetosomes) in magnetotactic bacteria are arranged in straight chains. After isolation from these bacteria those chains tend to form closed loops. The reason for these phenomena is that the existence of lipid membrane surrounding magnetic core prevents them to stick together by electrostatic repulsion [4]. As was stated earlier, elasticity may play a major role in the magnetosome arrangement of the bent configuration [5]. The presence of narrow shape and size distribution for magnetite crystals (magnetosomes) within magnetotactic bacteria suggests strongly that there are species-specific mechanisms that control the process of biomineralization.

The present paper reports on detailed hysteresis and remanent magnetization measurements from $2 \mathrm{~K}$ up to room temperature on magnetosomes with special attention to the behavior at the Verwey transition.

\section{Materials and methods}

Bacterial magnetosomes investigated in this contribution were synthesized by magnetotactic bacteria Magnetospirillum sp. strain AMB-1 in laboratory conditions. This bacteria is a Gram-negative $\alpha$-proteobacterium that is more oxygen-tolerant and easier to grow on a large scale. The detailed description of the cultivation process of magnetotactic bacteria and the isolation of magnetosomes from bacteria is given in our previous contribution [6]. Owing to the presence of the enveloping membrane, isolated magnetosome particles form stable, well-dispersed suspensions in water solution of HEPES (4-(2-hydroxyethyl)-1-piperazineethanesulfonic acid). Typically, we were able to obtain $2.6 \mathrm{mg}$ magnetosomes from a $1000 \mathrm{~mL}$ bacterial culture.

Atomic force microscopy (AFM) experiments in this paper were performed with the Dimension 3100 atomic force microscope retrofitted with the Nanoscope V controller (Vecco, Digital Instruments, Santa Barbara, CA, USA). Images were obtained in the tapping mode with the super sharp silicone tip (MikroMasch DP15/HI'RES/AIBS, Estonia), having less than $1 \mathrm{~nm}$ 
in radius, resonance frequency of about $325 \mathrm{MHz}$, and spring constant of about $46 \mathrm{~N} / \mathrm{m}$. In the tapping mode regime, the AFM cantilever oscillates close to its resonance frequency and the tip only slightly touches the surface. All measurements were done in air (humidity of about $30 \%$ ) at room temperature and a scan rate of $1 \mathrm{~Hz}$. Samples of magnetosomes in HEPES AFM investigation were prepared by spin coating (SC) deposition and droplet deposition on Gritek polished silicon wafers (GRINM Semiconductor Materials Co., Ltd., China) and high-grade mica wafers (Ted Pella, Inc., CA, USA), respectively. Preceding the magnetosomes deposition by SC technology, the silicone wafers were cleaned up by immersion in the dichloromethane for about $10 \mathrm{~min}$. For improvement of hydrophobicity, the substrates were made hydrophilic in water (double purified MilliQ water, $18 \mathrm{M} \Omega \mathrm{cm}^{-1}$ ), ammonia and hydrogen peroxide solution by immersion for about $20 \mathrm{~min}$ at $80^{\circ} \mathrm{C}$. Mica substrates were freshly prepared immediately before droplet deposition by cleavage. The studied magnetosomes were highly diluted in HEPES to reduce the viscosity of the liquid. To generate very thin films, in the SC process the rotation speed of $1500 \mathrm{rpm}$ was applied (acceleration phase of $500 \mathrm{rpm} \mathrm{s}^{-1}$, deceleration phase can be negligible). The rotation time $(60 \mathrm{~s})$ facilitated the magnetosomes dispersion effect and support the evaporation of the solution. The nanoparticles were then fixed to the silicone substrates. One drop (about $1 \mu \mathrm{L}$ ) was dropped on mica substrates. The liquid was then spread over surface via its excellent hydrophilic properties. Finally, all samples were placed in an oven for $12 \mathrm{~h}$ immediately after depositions at temperature of $30^{\circ} \mathrm{C}$ and pressure of about 20 mbar to remove remaining solvent in the films.

A Quantum Design MPMS superconducting quantum interference device magnetometer was used for magnetic measurements reported here. The remanent magnetization line was determined from temperature dependence (2-270 K) during warming process (RMW) after turning off field-cooling (FC) magnetic field (500 Oe) when temperature reached $2 \mathrm{~K}$. The annealing of this metastable remanent magnetization can provide useful information regarding barrier and anisotropy. The coercive force was obtained from hysteresis loops measurements at various temperatures in magnetic fields up to $5 \mathrm{~T}$.

\section{Results}

According to our previous article [6], the X-ray diffraction (XRD) using Co $K_{\alpha}$ radiations with a wavelength of $\lambda=0.17903 \mathrm{~nm}$ has shown that powder diffraction peaks of magnetosomes well fit with standard $\mathrm{Fe}_{3} \mathrm{O}_{4}$ reflections which reveals that the magnetic nanocrystals within the magnetosome consisted of magnetite without another phases. From the line-broadening of 311 peak of XRD powder diffraction pattern using the Scherrer equation the magnetosome diameter was calculated to be $37 \mathrm{~nm}$. On the other side, as we have stated previously [6] and [7], the micrographs estimated from transmission electron microscopy (TEM) using JEOL 1200EX
Microscope working at $120 \mathrm{kV}$ and $80000 \times$ magnification by replication technique, showed that in our sample the magnetosomes after isolation are arranged in chains with tendency to create the bent and closed loops as a consequence of minimizing their magnetic stray field energy. This arrangement of chains with 6-12 magnetosomes allows the existence of lipid membrane surrounding magnetic core which prevents them to stick together by electrostatic repulsion [5]. The mean size and standard deviation estimated from TEM was $34 \mathrm{~nm}$ and $6 \mathrm{~nm}$, respectively.

Figure 1 presents AFM topography image of studied magnetosomes deposited by spin coating. It was found that magnetosomes distributed on silicone and mica form clusters as well as single, isolated magnetite particles of various sizes. Due to capillary forces (especially during solvent evaporation) and magneto-dipole-dipole interactions clusters of few and many magnetosomes are mainly formed [8]. The geometrical sizes of magnetosomes (Fig. 1) were measured to be of about 20-100 nm (in plane parallel to the silicon or mica surface), respectively. Moreover, based on AFM images of separated magnetosomes (Fig. 1, right), it seems that their shape is more ellipsoidal than spherical [9] which can contribute to the shape anisotropy of sample. The height and the lateral diameter measured by means of AFM do not correspond to each other, since such a cluster is formed. From analysis image (Fig. 1, right) the diameter of chosen magnetosome was estimated to be $54.8 \mathrm{~nm}$ which is higher value as mean diameter estimated from TEM experiments which can be related to the various techniques of measurement.

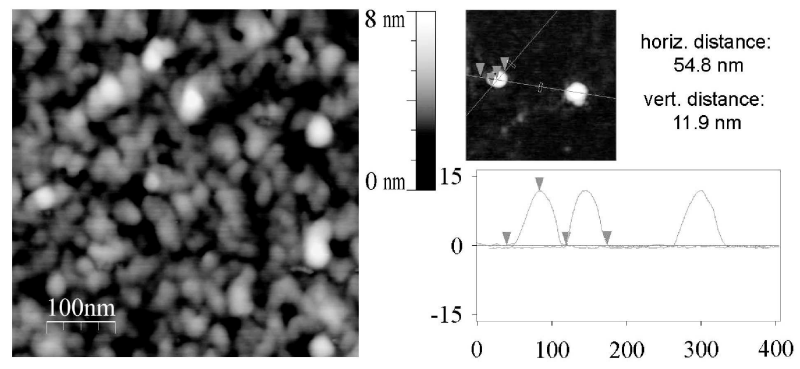

Fig. 1. AFM height image of magnetosomes deposited by spin coating on silicone wafers (left) and section analysis (right) image (axes in $\mathrm{nm}$ ).

In our previous work [6] the field cooling and zero field cooling measurements of magnetization showed the sharp changes of magnetization in the vicinity of the Verwey transition $(\approx 105 \mathrm{~K})$. It seems that the best way to reveal the magnetic signature of the Verwey transition is to measure the temperature dependence of remanent magnetization (RMW) obtained after switching off the magnetic field applied during the field cooling experiment as this provides useful information regarding to the barriers and anisotropy in sample. In this case the thermal energy $k_{\mathrm{B}} T$ only competes with 
the dipolar collective barriers and magnetocrystalline anisotropy. The temperature dependences of the coercive force and remanent magnetization measured by MPMS instrument above described process are given in Fig. 2. In temperature dependence curve of the

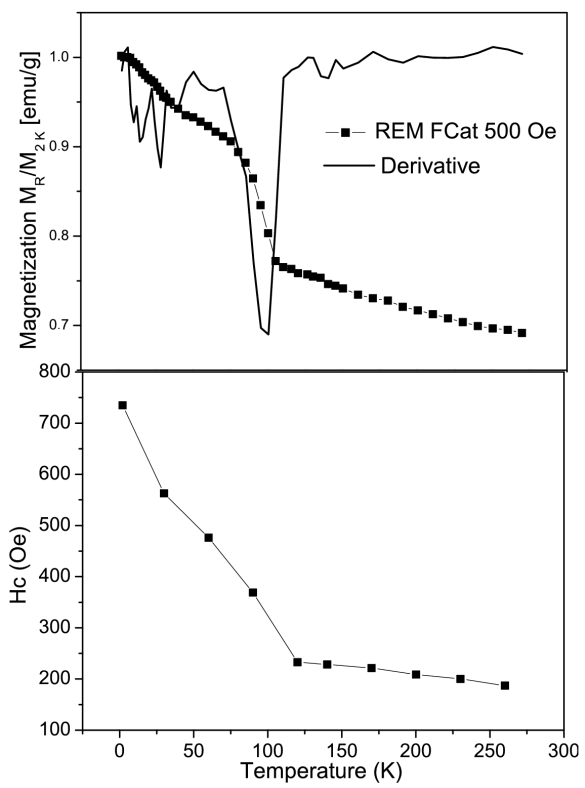

Fig. 2. Temperature dependence of the remanent magnetization after switching off magnetic field of FC (500 Oe). The solid line shows the derivative of RWM curve (top). Coercive fields vs. temperature estimated from hysteresis loops measured for temperature below and above the Verwey transition (bottom).

remanent magnetization a major drop due to the Verwey transition was observed between 90 and $110 \mathrm{~K}$ (Fig. 2, top) which is clearly shown in the derivative line of the RMW. Hysteresis loops were measured in a maximum field of $5 \mathrm{~T}$ at selected temperatures from $2 \mathrm{~K}$ to $270 \mathrm{~K}$ using SQUID magnetometer. Values of coercive forces at chosen temperatures are plotted in Fig. 2 (bottom). Coercive force is practically temperature independent above the Verwey transition $(105 \mathrm{~K})$ and increases by factor of 4 below this transition. The increase in $H_{\mathrm{c}}$ in the vicinity of $T_{\mathrm{v}}$ has been observed previously [3] for submicron magnetite nanoparticles with medium particle sizes $\leq 0.22 \mu \mathrm{m}$. This increase reflects the large increase in magnetocrystalline anisotropy in the cubicmonoclinic phase transition. The ratio of remanent and saturation magnetization $\left(M_{\mathrm{r}} / M_{\mathrm{S}}\right)$ was estimated to be 0.47 is in excellent agreement with the theoretical value of 0.5 for a random dispersion of single-magnetic-domain particles. As it was stated in case of small nanoparticles the transition is smeared and remanent magnetization and $H_{\mathrm{c}}$ are smaller. But in the case of magnetosomes the chains act as long dipoles with enhanced effective anisotropy along the chain and thermal energy is insufficient to overcome this barrier [10]. Therefore we can conclude that for magnetization behavior, large effective anisotropy coming from chains is responsible.

\section{Conclusion}

In this work we have studied the bacterial magnetite nanoparticles (magnetosomes) prepared by mineralization process. The AFM experiment showed that the shape of individual magnetosomes is more ellipsoidal than spherical and shape anisotropy can contribute to the character of transition, too. The sharp transition in temperature dependence of remanent magnetization and coercive force at the Verwey transition $(105 \mathrm{~K})$ is connected with existence of chains of magnetosomes that act as long dipoles with enhanced effective anisotropy along these chains.

\section{Acknowledgments}

The Slovak Academy of Sciences, within the framework of Project VEGA No.6166 and Slovak Research and Development Agency, within the framework of Projects APVV No.APVV-0173-06, APVV 0509-07 and APVV99-026505, supported this work.

\section{References}

[1] E.J.W. Verwey, Nature (London) 144, 327 (1939).

[2] A. Kosterov, Earth Planet. Sci. Lett. 186, 245 (2001).

[3] Ö. Özdemir, D.J. Dunlop, B.M. Moskowitz, Earth Planet. Sci. Lett. 194, 343 (2002).

[4] D. Schüler, J. Mol. Microbiol. Biotechnol. 1, 79 (1999).

[5] P. Valeri, J. Scherbakov, M. Winklhofer, Eur. Biophys. J. 26, 319 (1997).

[6] M. Timko, A. Džarová, V. Závišová, M. Koneracká, A. Šprincová, P. Kopčanský, J. Kováč, I. Vávra, A. Szlaferek, Magnetohydrodynamics 44, 3 (2008).

[7] M. Timko, A. Džarová, P. Kopčanský, V. Závišová, M. Koneracká, A. Sprincová, M. Vaclavíková, L. Ivaničová, I. Vávra, Acta Phys. Pol. A 113, 573 (2008).

[8] K.R. Reddy, K.P. Lee, A.G. Iyengar, J. Appl. Polymer Sci. 104, 4127 (2007).

[9] M. Rasa, A.P. Philipse, J. Magn. Magn. Mater. 252, 101 (2002).

[10] R. Prozorov, T. Prozorov, S.K. Mallapragada, K. Surya, B. Narasimhan, T.J. Williams, D.A. Bazylinski, Phys. Rev. B 76, 054406 (2007). 